
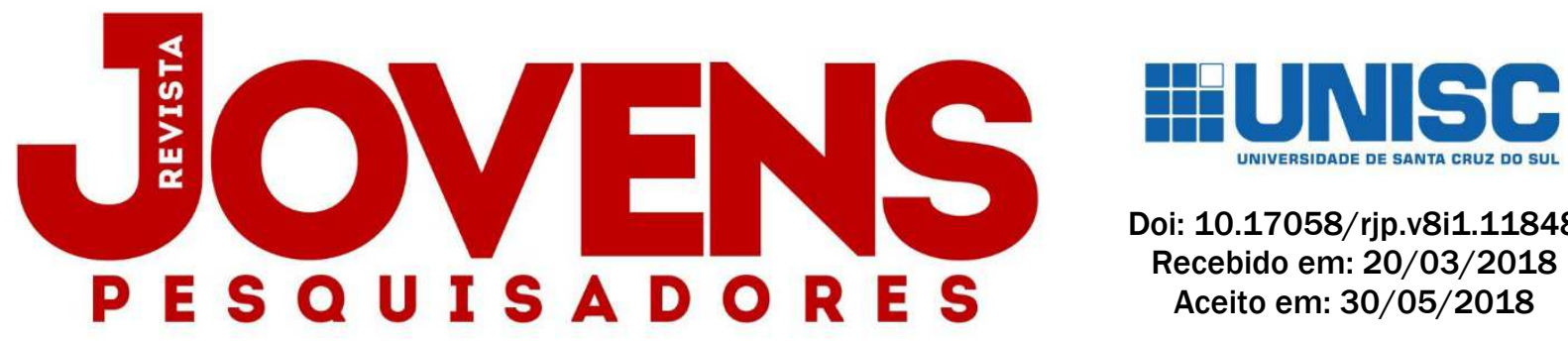

Doi: $10.17058 /$ rjp.v8i1.11848

Recebido em: 20/03/2018

Aceito em: 30/05/2018

\title{
UMA ABORDAGEM SOBRE A DETERMINAÇÃO \\ DE ATIVIDADE ANTIOXIDANTE E FENÓIS TOTAIS POR FT-IR E QUIMIOMETRIA EM ALIMENTOS
}

SOUZA, P. B. ${ }^{1}$; PEREIRA, P. H. M. ${ }^{2}$; ROHLFES, A. L. B. ${ }^{3}$; BACCAR, N. M. ${ }^{3}$; MARQUARDT, L.4; CORBELLINI, V. A. ${ }^{3}$

\author{
PALAVRAS CHAVE: Alimentos. Nutracêuticos. FT-IR. Quimiometria.
}

\begin{abstract}
RESUMO
Os componentes bioativos presentes nos alimentos são conhecidos por suas propriedades nutracêuticas sendo necessário desenvolver metodologias analíticas capazes de determinar e quantificar tais compostos. No entanto, os métodos disponíveis para tais análises que são essencialmente baseados em reações colorimétricas, demandam tempo e implicam em considerável geração de custos, decorrente do alto valor dos reagentes, e de resíduos. Com isso, as técnicas moleculares como a Espectroscopia de Absorção Molecular no Infravermelho com transformada de Fourier (FTIR) aliada à quimiometria surgem como técnicas complementares para a análise de dados multivariados provenientes das amostras por meio de modelos de predição. Esta revisão aborda uma atualização referente ao período de 2013 a 2018 no uso de modelos quimiométricos como complemento à análise de atividade antioxidante e conteúdo fenólico total em alimentos sólidos. Em razão de a técnica ser propícia para atender aos requisitos de consciência ambiental e economia de custos sem prejuízo nos resultados de pesquisa, verifica-se a importância do desenvolvimento de métodos à base de FT-IR associados a recursos quimiométricos para análise de componentes alimentares.
\end{abstract}

\section{AN APPROACH ON THE DETERMINATION OF ANTIOXIDANT ACTIVITY AND TOTAL PHENOLS BY FT-IR AND CHEMOMETRICS IN FOODS}

\author{
KEYWORDS: Food. Nutraceutical. FT-IR. Chemometrics.
}

\begin{abstract}
The bioactive compounds present in foods are known for their nutraceutical properties and it's necessary to develop analytical methodologies capable of determining and quantifying such compounds. However, the methods available for such analyzes require time and imply considerable cost, due to the high value of the reagents, and waste generation. Therefore, molecular techniques such as Fourier transform Infrared Molecular Absorption Spectroscopy (FT-IR) allied to chemometric appear as complementary techniques for the analysis of multivariate data from the samples by means of prediction models. This review adresses an update from 2013 to 2018 in the use of chemometric models as a complement to the analysis of antioxidant activity and total phenolic content and properties in solid foods. Because the technique is propitious to meet the requirements of environmental awareness and cost saving without prejudice to the results of research, the importance of development of FT-IR based methods associated to chemometic resources for analysis of food components is verified. Keywords: Food. Nutraceutical. FT-IR. Chemometrics.
\end{abstract}

\footnotetext{
${ }^{1}$ Acadêmica do curso de Farmácia na Universidade de Santa Cruz do Sul.

2 Acadêmico do curso de Química na Universidade de Santa Cruz do Sul.

3 Docentes do Departamento de Química e Física na Universidade de Santa Cruz do Sul.

4 Docente do Departamento de Engenharia, Arquitetura e Ciências Agrárias na Universidade de Santa Cruz do Sul.
} 


\section{INTRODUÇÃO}

A reconhecida importância da ingestão de compostos bioativos (WILSON D. W. et al., 2017; HUANG D., 2018; RAMIREZ-EXPOSITO M. J; MARTINEZ-MARTOS J. M., 2018) torna oportuno determinar em quais alimentos tais compostos estarão presentes. Para isso foram desenvolvidos métodos analíticos baseados em reações colorimétricas para determinação da atividade antioxidante e determinação de fenóis totais em alimentos (VÁZQUEZ C. V. et al., 2015).

Com o avanço das técnicas moleculares, inclusive no campo da ciência dos alimentos, foram elaborados métodos instrumentais baseados em radiação eletromagnética como a Espectroscopia de Absorção Molecular no Infravermelho com transformada de Fourier (FT-IR) que tem sido utilizada desde a década de 60 para análise de alimentos (CADET F; GARRIDES S.; GUARDIA M., 2012). A região do espectro eletromagnético situada no comprimento de onda de 4000 a 650 (700) $\mathrm{cm}^{-1}$ é denominada região do infravermelho médio e tem sido utilizada nessa investigação (FRAGOSO S. et al. 2011; GODIM C. S. et al., 2017; NHOUCHI Z; KAROUI R., 2018).

A observação técnica do espectro gerado pelos equipamentos de FT-IR proporciona, por si só, a descoberta dos grupamentos químicos que compõem a amostra (SVEČNJAK, L. et al., 2017). Adicionalmente, alia-se a análise por FT-IR à análise multivariada de dados, tornando possível a construção de modelos quimiométricos para a predição de uma propriedade em determinado alimento (SIVAM A. S. et al., 2011; HU Y. et al, 2016).

Embasada nessas informações, esta revisão aborda os métodos adotados para determinação de atividade antioxidante e de fenóis totais em alimentos no contexto da química analítica convencional. Conjuntamente, são apresentados aspectos de funcionamento do equipamento de FT-IR, assim como aplicações da técnica associada à quimiometria para estimar a presença de compostos bioativos nesse tipo de amostra.

\section{METODOLOGIAS}

\subsection{MÉTODO PADRÃO PARA DETERMINAÇÃO DA ATIVIDADE ANTIOXIDANTE}

0 método de captura de radicais DPPH (1,1-difenil-2-picrilidrazil) é amplamente utilizado na determinação da atividade antioxidante de compostos ou extratos específicos em matrizes alimentares (OTHMAN S. B. et al., 2015; SOQUETTA M. B., 2016; IRONDI E. A. et al., 2017). A quantificação dessa propriedade dá-se por meio do monitoramento da descoloração das soluções contendo o radical livre estável DPPH e a amostra, com o uso de espectrofotômetro em comprimento de onda característico (BRAND-WILIAMS, W.; CUVELIER, M.E.; BERSET, C., 1995). DPPH é um radical livre estável, que ao aceitar um elétron ou um radical de hidrogênio fornecido por antioxidantes 
presentes no meio reacional passa a ser uma molécula diamagnética estável (BRAND-WILIAMS, W.; CUVELIER, M.E.; BERSET, C., 1995). A atividade antioxidante é calculada em porcentagem, em equivalentes do antioxidante utilizado como padrão ou ainda pelo parâmetro $\mathrm{EC}_{50}$ que corresponde à concentração de compostos redutores necessária para reduzir à metade a quantidade de radicais livres DPPH (BREWER L. R. et al, 2014; (LOHANI U. C.; MUTHUKUMARAPPAN K.; MELETHARAYIL G. H., 2016; ALVES S. A. et al., 2017). Existem variações metodológicas como o uso de sistemas de injeção de fluxo associados a eletrodos modificados com DPPH ou outras técnicas eletroquímicas como a amperometria (MILARDOVIČ S.; BOŽIDAR G. S.; IVEKOVIČ D., 2006; ZIYATDINOVA G.; SNEGUREVA Y; BUDNIKOV H., 2017). No entanto, por se tratar de radical centrado no nitrogênio, há peculiaridades em sua reação com antioxidantes, dando preferência a pequenos compostos reativos, subestimando os antioxidantes de moléculas grandes (IUPAC, 2013).

\subsection{MÉTODO PADRÃO PARA DETERMINAÇÃO DE FENÓIS TOTAIS}

Um método tradicionalmente utilizado para determinação de fenóis totais em alimentos emprega o reagente fenol de Folin Ciocalteau, composto essencialmente por tungstato e molibdato (FOLIN O.; CIOCALTEU V., 1927; TURKMEN N.; SARI F.; VELIOGLU S., 2006; PANDEY P.; AWASTHI P., 2015). 0 molibdênio e o tungstênio estão no estado de oxidação 6+ que na presença de agentes redutores, como antioxidantes e compostos fenólicos, em meio alcalino, formam o complexo [(PMoW $\left.\left.{ }_{11} \mathrm{O}_{4}\right)^{-4}\right]$ de coloração azul. Desta forma o ensaio fundamenta-se no monitoramento do decréscimo da absorbância das soluções testadas e correlaciona-se linearmente com a diminuição da absorbância com a quantidade de substâncias redutoras presentes na amostra teste (SINGELTON V. L.; ROSSI Jr. J. A., 1965; MARGRAF T. et al, 2015). Foram propostas adaptações no método como o uso de microplacas com monitoramento contínuo da absorbância proporcionando a simplificação do ensaio e a redução do consumo de reagentes e da geração de resíduos (MAGALHÃES L. M. et al., 2010). Embora seja utilizado para essa determinação, o método baseia-se em uma reação de oxirredução e, assim, pode ser apontado como um método para determinação da atividade antioxidante. Além disso, o método sofre interferências devido à reatividade com outras substâncias como açúcares, aminas aromáticas e Fe II o que pode superestimar o conteúdo fenólico da amostra (PRIOR R.; WU X.; SCHAICH K., 2005). Suas aplicações não são restritas a amostras alimentícias tendo inclusive sido usado na determinação de agentes anti-inflamatórios em preparações farmacêuticas (SASTRY C. S. P.; RAO A. R. M., 1988). 


\subsection{FT-IR ASSOCIADA À QUIMIOMETRIA PARA ANÁLISE DE ALIMENTOS}

A FT-IR caracteriza-se como uma técnica não destrutiva de amostras (OLSEN E. F. et al., 2008). Um espectrofotômetro de FT-IR é constituído conforme ilustração simplificada na figura 1, por uma fonte de luz responsável pela produção do feixe de irradiação infravermelha, o interferômetro pelo qual passa o feixe para ocorrer a codificação do espectro, o interferograma que constitui interferência destrutiva e construtiva oriunda da recombinação de feixes com diferentes caminhos no interferômetro. A partir daí, o feixe entra em contato com a amostra e esta absorve frequências específicas de energia características daquele material. Em seguida, o detector mensura o sinal de interferograma versus tempo para todas as frequências simultaneamente. No entanto, sobrepõe-se um feixe que servirá como referência ou fundo para a operação do instrumento. Ao final, obtêm-se o espectro assim que o interferograma subtraia involuntariamente o espectro de fundo do espectro da amostra mediante software de transformação de Fourier (MOHAMED M. A., 2017).

Figura 1: Imagem ilustrativa simplificada dos componentes do espectrofotômetro FT-IR.

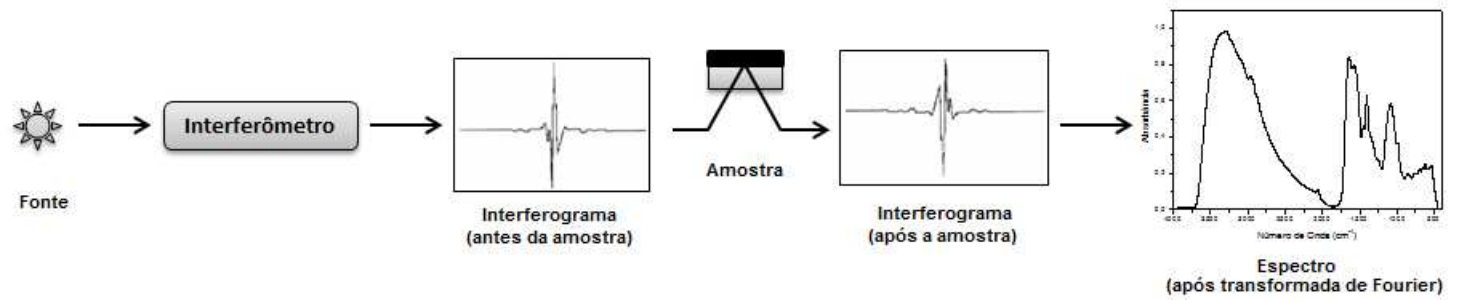

Fonte: Autor.

Essa técnica vem sendo explorada para elucidação da estrutura molecular de determinada amostra fundamentada no conhecimento que duas estruturas químicas não apresentarão os mesmos espectros FT-IR (YAP K.Y.L; CHAN S. Y.; LIM C. S., 2007).

A análise por FT-IR é sucedida pela análise estatística dos seus resultados. Ao trabalhar com inúmeras variáveis em um sistema analítico, torna - se necessária a análise multivariada de dados que abrange a quimiometria, utilizando algoritmos para a construção de modelos de predição (LOPES J. A. et al., 2004; WU Z., et al, 2016; ALEIXANDRE-TUDO J. L. et al., 2018).

\section{METODOLOGIA}

Para a elaboração deste trabalho foram consultados periódicos indexados nas bases de dados Science Direct, Scopus, Springer Link e Wiley Online Library. Utilizando diversas estratégias de busca, compreendendo os últimos cinco anos, foram priorizados os trabalhos que tratavam do uso de modelos quimiométricos à base de FT-IR, região do infravermelho médio, para predição de atividade antioxidante e conteúdo fenólico total em amostras alimentícias. Como critério de exclusão foram 
desprezados os artigos que não mencionavam o uso de modelagem quimiométrica por FT-IR para quantificação de propriedades bioativas ou físico-químicas em alimentos sólidos. Em contrapartida, atendendo aos critérios opostos foram encontrados 5 trabalhos.

\section{RESULTADOS E DISCUSSÃO}

A fim de ilustrar a relevância estatística de cada estudo revisado, foi construída a Tabela 1, que contempla a técnica e as variáveis quimiométricas com esclarecimentos sobre amostra, técnica, método estatístico multivariado e resultados de cada determinação analítica.

Tabela 1: Características destacadas nos estudos revisados.

\begin{tabular}{|c|c|c|c|c|c|c|}
\hline Amostra & $\begin{array}{l}\text { Número } \\
\text { Total de } \\
\text { Amostras } \\
(\mathrm{n})\end{array}$ & Técnica & $\begin{array}{c}\text { Método } \\
\text { Estatístico } \\
\text { Multivariado }\end{array}$ & Resultados & Observações & Autor \\
\hline Framboesa & 34 & $\begin{array}{c}\text { FT-IR, } \\
\text { faixa de } \\
5020 \text { a } \\
925 \mathrm{~cm}^{-1}, \\
4 \mathrm{~cm}^{-1} \\
\text { resolução } \\
\text { espectral }\end{array}$ & PCA; PLS & $\begin{array}{l}\text { O algoritmo PLS foi } \\
\text { desenvolvido com o } \\
\text { uso de } 12 \text { variáveis } \\
\text { latentes (VL); } \\
\text { Coeficiente de } \\
\text { determinação }\left(\mathrm{R}^{2}\right)= \\
0,86, \text { para predição de } \\
\text { atividade antioxidante } \\
\text { e de } 0,72 \text { para } \\
\text { predição do teor de } \\
\text { fenóis totais; Erro } \\
\text { quadrático médio de } \\
\text { validação (RMSEV) = } \\
\text { 18,6 e } 70,82 . \text { Entre os } \\
\text { modelos construídos } \\
\text { pelo autor, foi } \\
\text { considerada a } \\
\text { correlação com a } \\
\text { determinação do teor } \\
\text { de fenóis totais, } \\
\text { expressos em } \\
\text { GAE/100g de } \\
\text { amostra, de acordo } \\
\text { com metodologia de } \\
\text { referência. }\end{array}$ & $\begin{array}{l}\text { Foram realizadas duas } \\
\text { validações, diferenciadas } \\
\text { pelo tempo de um ano } \\
\text { entre uma e outra. Para } \\
\text { análise foi utilizado o } \\
\text { extrato aquoso obtido da } \\
\text { polpa e casca de } \\
\text { diferentes cultivares do } \\
\text { fruto. }\end{array}$ & $\begin{array}{c}\text { (Z. N, } \\
\text { Andrjanjaka- } \\
\text { Camps et al., } \\
\text { 2015) }\end{array}$ \\
\hline $\begin{array}{c}\text { Grãos de } \\
\text { café }\end{array}$ & 42 & $\begin{array}{c}\text { FT-IR ATR, } \\
\text { faixa de } \\
4000 \mathrm{a} \\
400 \mathrm{~cm}^{-1} ; \\
2 \mathrm{~cm}^{-1} \mathrm{de} \\
\text { resolução } \\
\text { espectral; } \\
16 \text { scans }\end{array}$ & PLSR & $\begin{array}{c}\text { Em modelo } \\
\text { quimiométrico obtido } \\
\text { a partir da correlação } \\
\text { com os resultados } \\
\text { para atividade } \\
\text { antioxidante expressos } \\
\text { em } \mu \mathrm{M} \text { Trolox/g de } \\
\text { amostra, foi obtido } \\
\mathrm{R}^{2}=0,811 \text { e RMSEV }= \\
14,392\end{array}$ & $\begin{array}{c}\text { As amostras foram } \\
\text { pulverizadas para análise } \\
\text { por FT-IR. }\end{array}$ & $\begin{array}{c}\text { (Liang, Ningjian } \\
\text { et al., 2016) }\end{array}$ \\
\hline
\end{tabular}




\begin{tabular}{|c|c|c|c|c|c|c|}
\hline $\begin{array}{c}\text { Farinha de } \\
\text { arroz }\end{array}$ & 50 & $\begin{array}{l}\text { FT-IR ATR } \\
\text { com } \\
\text { detector } \\
\text { MCT, faixa } \\
\text { de } 4000 \text { a } \\
400 \mathrm{~cm}^{-1} ; \\
4 \mathrm{~cm}^{-1} \text { de } \\
\text { resolução; } \\
64 \text { scans }\end{array}$ & $\begin{array}{l}\text { DFA; PCA; } \\
\text { PLSR }\end{array}$ & $\begin{array}{c}\text { Foram construídos } \\
\text { modelos } \\
\text { quimiométricos tanto } \\
\text { para determinação de } \\
\text { fenóis totais (mg } \\
\text { GAE/g) quanto para } \\
\text { determinação de } \\
\text { atividade antioxidante } \\
\text { (mM TE/g) em } \\
\text { farinhas de quatro } \\
\text { variedades de arroz } \\
\text { cru/cozido, } \\
\text { considerando somente } \\
\text { a região espectral de } \\
\text { 1800 a } 900 \mathrm{~cm}-1 . \\
\text { R2=0,9715;0,9359/0, } \\
8933 ; 0,8933 \text {. RMSEV: } \\
\text { Atividade } \\
\text { antioxidante/Fenois } \\
\text { totais: } 0,010 / 0,230 \\
\text { (Arroz preto cru); } \\
0,009 / 0,241 \text { (arroz } \\
\text { vermelho cru); } \\
0,011 / 1,015 \text { (arroz } \\
\text { castanho cru); } \\
0,027 / 0,451 \text { (arroz } \\
\text { branco cru); } \\
0,219 / 0,086 \text { (arroz } \\
\text { preto cozido); } \\
0,175 / 0,372 \text { (arroz } \\
\text { vermelho cozido); } \\
0,107 / 0,079 \text { (arroz } \\
\text { castanho cozido); } \\
0,098 / 0,913 \text { (arroz } \\
\text { branco cozido). }\end{array}$ & $\begin{array}{c}\text { As amostras foram } \\
\text { diretamente colocadas } \\
\text { sobre o cristal de HATR. }\end{array}$ & $\begin{array}{c}\text { (Jantasee, } \\
\text { Ampawan et al., } \\
\text { 2013) }\end{array}$ \\
\hline $\begin{array}{c}\text { Farinha de } \\
\text { quinoa }\end{array}$ & 65 & $\begin{array}{c}\text { FT-IR ATR, } \\
4000 \mathrm{a} \\
300 \mathrm{~cm}^{-1} ; \\
4 \mathrm{~cm}^{-1} \mathrm{de} \\
\text { resolução; } \\
16 \text { scans }\end{array}$ & PLS & $\begin{array}{c}\text { Em modelo } \\
\text { quimiométrico para } \\
\text { predição da umidade } \\
\text { em amostras de } \\
\text { farinha de quinoa, } \\
\text { considerando a região } \\
\text { espectral entre } 3000 \text { e } \\
3600 \mathrm{~cm}^{-1} \\
\text { (relacionada ao } \\
\text { grupamento - OH) } \\
\text { (Che Man, Y.B.; } \\
\text { Mirghani, M.E.S., } \\
\text { 2000). Foi obtido R } \text { R }^{2}= \\
0,872 \text {. }\end{array}$ & $\begin{array}{l}\text { Para análise, foram } \\
\text { preparadas cápsulas de } \\
\text { diferentes teores de } \\
\text { umidade a partir da } \\
\text { amostra. O material foi } \\
\text { colocado sobre o cristal } \\
\text { de ATR. Os autores } \\
\text { citaram 100\% de } \\
\text { previsibilidade para as } \\
\text { equações propostas, não } \\
\text { informando o parâmetro } \\
\text { RMSEV, correspondente } \\
\text { à etapa de validação } \\
\text { cruzada. }\end{array}$ & $\begin{array}{c}\text { (González } \\
\text { Muñoz, Àdrian et } \\
\text { al., 2016) }\end{array}$ \\
\hline Chocolate & 13 & $\begin{array}{l}\text { FT-IR ATR, } \\
4000 \text { a } \\
550 \mathrm{~cm}^{-1} ; \\
2 \mathrm{~cm}^{-1} \text { de } \\
\text { resolução; } \\
16 \text { scans }\end{array}$ & PLSR & $\begin{array}{c}\text { O modelo } \\
\text { quimiométrico para } \\
\text { predição da atividade } \\
\text { antioxidante de acordo } \\
\text { com o método DPPH } \\
\text { apresentou } \\
\text { RMSEV=10,87 } \mu \text { mol } \\
\text { Trolox/g de chocolate } \\
\text { desengordurado. Foi } \\
\text { obtido } \mathrm{R}^{2}=0,89 ; \text { No } \\
\text { modelo desenvolvido } \\
\text { para predição de } \\
\text { fenóis totais pelo } \\
\text { método de Folin } \\
\text { Ciocalteu foi } \\
\text { encontrado RMSEV= }\end{array}$ & $\begin{array}{c}\text { Para construção do } \\
\text { modelo foi utilizada a } \\
\text { região espectral de } 1800 \\
\text { a } 700 \mathrm{~cm}^{-1} \text { devido } \\
\text { aos grupos funcionais de } \\
\text { maior interesse situarem } \\
\text { - se nessa região. Para } \\
\text { obter espectro padrão foi } \\
\text { utilizada a catequina em } \\
\text { pó. }\end{array}$ & $\begin{array}{c}\text { (Hu, Yaxi et al, } \\
\text { 2016) }\end{array}$ \\
\hline
\end{tabular}




\begin{tabular}{|l|l|l|l|l|l|}
\hline & & & $\begin{array}{c}4,21 \mathrm{mg} \mathrm{GAE} / \mathrm{g} \text { de } \\
\text { chocolate } \\
\text { desengordurado e } \\
\mathrm{R}^{2}=0,88 .\end{array}$ & & \\
\hline
\end{tabular}

Considerando-se que há diversos estudos em que os pesquisadores utilizam FT-IR para elucidação estrutural da amostra (BHAT R.; SHAHARUDDIN N. A.; KUANG Y. T., 2013; VALENCIA A. G. et al., 2015; RAMACHANDRAIAH K.; CHIN B. K., 2017) foi encontrada quantidade limitada de publicações nas quais se desenvolveu modelo quimiométrico a partir da análise por FT-IR e do método tradicionalmente utilizado para análise da atividade antioxidante e conteúdo de compostos fenólicos. Essa realidade justifica a importância do desenvolvimento de tais ferramentas para futuros ensaios.

\section{CONSIDERAÇÕES FINAIS}

A FT-IR é uma ferramenta complementar vantajosa com relação aos métodos de química úmida, pois, dispõe do benefício de necessitar de pouca ou nenhuma preparação da amostra para análise. Dessa forma, a técnica é propícia para atender aos requisitos de consciência ambiental e economia de custos sem prejuízo nos resultados de pesquisa. Assim, verifica - se a importância do desenvolvimento de métodos à base de FT-IR associados a recursos quimiométricos para análise de componentes alimentares.

\section{REFERÊNCIAS}

ALEIXANDRE-TUDO, José Luis et al. Chemometric compositional analysis of phenolic compounds in fermenting samples and wines using different infrared spectroscopy techniques. Talanta, 176, 526536, 2018;

ALVES, A. S. et al. Physicochemical characterization and antioxidant activity of decolorized Moringa oleifera Lam leaf flour. Nutrire, 42, 31-35, 2017.

ANDRIANJAKA-CAMPS, Zo-Norosoa. Prediction of raspberries puree quality traits by Fourier transform infrared spectroscopy. LWT - Food Science and Technology, 63, 2, 1056-1062, 2015;

APAK, Reşat et al. Methods of measurement and evaluation of natural antioxidant capacity/activity (IUPAC Technical Report). Pure and Applied Chemistry, 85, 5, 957-998, 2013;

BHAT, Rajeev; SHAHARUDDIN, Nursyarfa Ayuni; KUANG, Yeoh Tow. A promising approach toward exploring nutritional and functional qualities of Beko (Oroxylum indicum L. BENTH. EX KURZ) pods for potential food applications. Journal of Food Processing and Preservation, 39, 1, 47-55, 2013;

BRAND-WILIAMS, Wendy; CUVELIER, Marie-Elisabeth.; BERSET, Claudette. Use of a free radical method to evaluate antioxidant activity. LWT - Food Science and Technology, 28, 1, 25-30, 1995;

BREWER, Lauren Renee et al. Wheat bran particle size influence on phytochemical extractability and antioxidant properties. Food Chemistry, 152, 483-490, 2014;

CADET, Frédéric; GARRIDES, Salvador.; GUARDIA, Miguel de la. Quantitative analysis, infrared. Encyclopedia of Analytical Chemistry, 2012.

FOLIN, Otto; CIOCALTEU, Vintila. On tyrosine and tryptophane determinations in proteins. The Journal of Biological Chemistry, 73, 2, 1927; 
FRAGOSO, Sandra et al. Quantification of phenolic compounds during red winemaking using FT-MIR spectroscopy and PLS-regression. Journal of Agricultural and Food Chemistry, 59, 20, 10795-10802, 2011;

GODIM, Carina de Souza et al. Detection of several common adulterants in raw milk by MID-infrared spectroscopy and one-class and multiclass multivariate strategies. Food Chemistry, 230, 68-75, 2017;

GONZÁLEZ MUÑOZ, Adrián et al. Rapid prediction of moisture content of quinoa (Chenopodium quinoa Willd.) flour by Fourier transform infrared (FTIR) spectroscopy Journal of Cereal Science, 71, 246-249, 2016;

$\mathrm{HU}$, Yaxi et al. Determination of antioxidant capacity and phenolic content of chocolate by attenuated total reflectance-Fourier transformed-infrared spectroscopy. Food Chemistry, 202, 254-261, 2016;

HUAND, Dejian. Dietary antioxidants and health promotion. Antioxidants (Basel), 7, 1, 9, 2018;

IRONDI, Emmanuel Anyachukwu et al. Effect of mango kernel flour addition on the phenolics profile, antioxidant activity and pasting properties of wheat flour. Journal of Food Measurement and Characterization, 11, 4, 2202-2210, 2017;

JANTASEE, Ampawan. et al. Fourier transform infrared spectroscopy for antioxidant capacity determination in colored glutinous rice. Food Analytical Methods, 7, 2, 389-399, 2014;

LIANG, Ningjian et al. Application of attenuated total reflectance-Fourier transformed infrared (ATRFTIR) spectroscopy to determine chlorogenic acid isomer profile and antioxidant capacity of coffee beans. Journal of Agricultural and Food Chemistry, 64, 3, 681-689, 2016;

LOHANI, Umesh C.; MUTHUKUMARAPPAN, Kasi; MELETHARAYIL, Gopinathan H. Application of hydrodynamic cavitation to improve antioxidant activity in sorghum flour and apple pomace. Food and Bioproducts Processing, 100, Part A, 335-343, 2016;

LOPES, João A. et al. Chemometrics in bioprocess engineering: process analytical technology (PAT) applications. Chemometrics and Intelligent Laboratory Systems, 74, 2, 269-275, 2004;

LU, Xionan; RASCO, Barbara. Determination of antioxidant content and antioxidant activity in foods using infrared spectroscopy and chemometrics: A Review. Critical Reviews in Food Science and Nutrition, 52, 10, 853-875, 2012;

MAGALHÃES, Luis M. et al. Rapid microplate high-throughput methodology for assessment of FolinCiocalteu reducing capacity. Talanta, 83, 2, 441-447, 2010;

MARGRAF, Thiago et al. Comparison between Folin-Ciocalteu and prussian blue assays to estimate the total phenolic content of juices and teas using 96-well microplates. Journal of Food Science, 80, 11, C2397-C2403, 2015;

MILARDOVIČ, Stjepan; BOŽIDAR, Grabarič; IVEKOVIČ, Damir. A novel amperometric method for antioxidant activity determination using DPPH free radical. Bioeletrochemistry, 68, 2, 175-180, 2006; MOHAMED, Mohamed Azuwa et al.. Fourier transform infrared (FTIR) spectroscopy. In Membrane Characterization. Elsevier Inc., 3-29, 2017;

NHOUCHI, Zeineb; KAROUI, Romdhane. Application of Fourier-transform mid infrared for the monitoring of pound cakes quality during storage. Food Chemistry, 252, 327-334, 2018;

OLSEN, Elisabeth Fjærvoll. et al. Determination of omega- 6 and omega-3 fatty acids in pork adipose tissue with nondestructive Raman and Fourier transform infrared spectroscopy. Applied Spectroscopy, 62, 9, 968-974, 2008;

OTHMAN, Sana Ben et al. Water-soluble extracts from defatted sesame seed flour show antioxidant activity in vitro. Food Chemistry, 175, 306-314, 2015;

PANDEY, Hemlata; AWASTHI, Pratima. Effect of processing techniques on nutritional composition and antioxidant activity of fenugreek (Trigonella foenum-graecum) seed flour. Journal of Food Science and Technology, 52, 2, 1054-1060, 2015;

PRIOR, Ronald L.; WU, Xianli; SCHAICH, Karen. Standarized methods for the determination of antioxidant capacity and phenolics in foods and dietary supplements. Journal of Agricultural and Food Chemistry, 53, 10, 4290-4302, 2005;

RAMACHANDRAIAH, Karna; CHIN, Koo Bok. Impact of drying and micronization on the physicochemical properties and antioxidant activities of celery stalk. Journal of Science Food and Agriculture, 97, 13, 4539-4547, 2017; 
RAMIREZ-EXPOSITO, María Jesús; MARTINEZ MARTOS, José Manuel. The delicate equilibrium between oxidants and antioxidants in brain glioma. Current Neuropharmacology, 16, 2018. Publicado on-line. Disponível em: https://doi.org/10.2174/1570159X16666180302120925. Acessado em: 08/03/18;

SASTRY, C. S. P.; RAO, A. Rama Mohana; Application of Folin Ciocalteu reagent for the spectrophotometric determination of some non steroidal antiinflamatory agents. Journal of Pharmacological Methods, 19, 2, 117-125, 1988;

SINGLETON V. L; ROSSI Jr Joseph A. Colorimetry of total phenolics with phosphomolybdicphosphotungstic acid reagent. American Journal of Enology Viticulture, 16, 3, 144-58, 1965;

SIVAM, A. S. et al. Application of FT-IR and Raman spectroscopy for the study of biopolymers in breads fortified with fibre and polyphenols. Food Research International, 50, 2, 574-585, 2013;

SOQUETTA, Marcela Bromberger et al. Characterization of physiochemical and microbiological properties and bioactive compounds, of flour made from the skin and bagasse of kiwi fruit (Actinidia deliciosa). Food Chemistry, 199, 471-478, 2016;

SVEČNJAK, Lidija et al. Characterization of Satsuma mandarin (Citrus unshiu Marc.) nectar-to-honey transformation pathway using FTIR-ATR spectroscopy. Food Chemistry, 232, 286-294, 2017.

TURKMEN, Nihal; SARI, Ferda; VELIOGLU, Sedat. Effects of extraction solvents on concentration and antioxidant activity of black and black mate tea polyphenols determined by ferrous tartrate and Folin Ciocalteu methods. Food Chemistry, 99, 4, 835-841, 2006;

VALENCIA, Germán Ayala et al. Physicochemical, morphological, and functional properties of flour and starch from peach palm (Bactris gasipaes K.) fruit. Starch, 67, 1-2, 163,173, 2015;

VÁSQUEZ, Cecilia Velázquez et al. Total phenolic compounds in Milk from different species. Design of an extraction technique for quantification using the Folin Ciocalteu method. Food Chemistry, 176, 480-486, 2015;

WILSON, Douglas W., et al. The role of food antioxidants, benefits of functional foods, and influence of feeding habits on the health of the older person: An overview. Antioxidants (Basel), 6, 4, 81, 2017;

WU, Zhengzong et al. Comparison between ATR-IR, Raman, concatenated ATR-IR and Raman spectroscopy for the determination of total antioxidant capacity and total phenolic content of Chinese rice wine. Food Chemistry, 194, 671-679, 2016;

YAP, Kevin Yi-Lwern.; CHAN, Sui Yung; LIM, Chu Sing. Infrared-based protocol for the identification and categorisation of ginseng and its products. Food Research International, 40, 5, 643-652, 2007;

ZIYATDINOVA, Guzel; SNEGUREVA, Yulia; BUDNIKOV, Herman. Novel approach for the voltammetric evaluation of antioxidant activity using DPPH -modified electrode. Eletrochimica Acta, 247, 97-106, 2017. 